\title{
Analysis of Sunspot Time Series During The Ascending Phase of Solar Cycle 24 Using The Wavelet Transform
}

\author{
Satish Kumar Kasde ${ }^{1, ~ *}$, Deepak Kumar Sondhiya ${ }^{2}$, Ashok Kumar Gwal ${ }^{1}$ \\ ${ }^{1}$ Space Science Research Laboratory, Department of Physics and Electronics, Barkatullah University, Bhopal, India \\ ${ }^{2}$ Department of Physics, LNCT Group of Colleges, Bhopal, India
}

\section{Email address:}

sbskasde@gmail.com (S. K. Kasde), deepsondhiya@gmail.com (D. K. Sondhiya), ashok.gwal@gmail.com (A. K. Gwal)

${ }^{*}$ Corresponding author

\section{To cite this article:}

Satish Kumar Kasde, Deepak Kumar Sondhiya, Ashok Kumar Gwal. Analysis of Sunspot Time Series During the Ascending Phase of Solar Cycle 24 Using the Wavelet Transform. American Journal of Modern Physics. Vol. 5, No. 5, 2016, pp. 79-86

doi: 10.11648/j.ajmp.20160505.11

Received: July 6, 2016; Accepted: July 18, 2016; Published: August 17, 2016

\begin{abstract}
The sunspots are widely used to measure the rotational rate of solar surface. We are interested in analysis of the temporal evaluation of the short-term period present in sunspot time series (i.e. sunspot number and area) during the ascending phase of Solar Cycle 24. For the better understanding of variation in solar activity originated at different layers of the solar atmosphere with respect to sunspot cycles, we study the phase relation between sunspot numbers and sunspot areas using cross correlation analysis techniques based on extended wavelet based approaches such as continuous wavelet transform, crosswavelet transform, and wavelet coherence. In this study we found the short-term periodicity " 27 days-rotational rate of Sun" for current solar cycle 24 (January 2008-May 2013), which suggested that the Solar Cycle 24 has minimum solar activity. We have also investigated the correlation between both parameters and identify the unusual conditions in space weather.
\end{abstract}

Keywords: Sunspots, Rotational Rate of Sun, Solar Cycle, Solar Activity, Wavelet Analysis

\section{Introduction}

The helioseismic revealed that the solar magnetic field is created at the base of convention zone (tachocline) through a dynamo mechanism. Because of buoyancy, this magnetic field rises in the upward direction and appears on the solar surface as sunspots, plages, and networks. In the long-term period, the Sun exhibits an early11-year sunspot cycle (Schwabe Cycle) and for short-term periods the 27-day periodicity is the most prominent [1], [2]. The 27 day periodicity revealed in the interval $1994-2000$ by [3]. The corresponding rotation period of the Sun found by [4] in their study of solar wind parameters of the interplanetary magnetic field (IMF). Several authors investigated the "midterm" periodicities of the sunspot data during Solar Cycles 12 through 23 [5]-[12]. Sunspot numbers and sunspot areas are historically used to describe the long-term, mid-term and short-term quasi periodicities. Mid-term quasi-periodicities (one to several months or longer) was used in the diagnostics of various solar flare activities and sunspot numbers or areas etc. [13]-[21], [5], [22], [5], [23]-[29]. The relation between total corrected sunspot area A (in millionths of the visible solar hemisphere) and sunspot number R is not particularly good for daily values, although it has often been stated that the relationship becomes better when using monthly or yearly averages.

This study aims to enhance the possibilities for detection and quantification of relationships between sunspot number and sunspot areas with wide stratigraphic uncertainties. Previous studies on this topic relied on basic statistical techniques such as cross-spectral analysis, regression, or correlation techniques. In addition, visual assessment of plots of such records is frequently used and extensively accepted [30]-[32]. Statistical methods and trend analysis are able to determine the significance of relationships between non-stationary time-series. However, performance of these methods compromised if time-series analyzed are same (i.e. having same wavelength) and phase shifted. Linear analysis approaches such as using the Fourier transform, may generate artifacts when they are applied to analysis of real world process. Currently, many advanced 
analysis approaches, such as the cross wavelet transform (XWT), wavelet coherence (WTC) and cross-recurrence plot (CRPs) are widely used to study the nonlinear behavior of time series [33].

Cross-wavelet analysis permits detection, extraction and re construction of relationships between two non-stationary signals simultaneously in frequency (or scale) and time (or location) [34]. Cross-spectral analysis is a bivariate version of spectral analysis for comparison of two data sets [35]. Similarly, cross-wavelet transform (XWT) can be considered as a bivariate extension to wavelet transform (WT), which has been developed as a tool to filter, exam, and extract nonstationary signals in time-series and images [36]. Arange of analysis approaches, namely continuous wavelet, cross wavelet, and wavelet coherence analyses, are employed to clarify the phase relationship between the smoothed monthly mean sunspot number and solar $10.7 \mathrm{~cm}$ flux (F10.7) [37]. Analysis shows that the raise region of high spectral power sitting across the Schwabe Cycle belt, where the two time series are in phase [38]. However, analysis of the crosswavelet transform and wavelet coherence unveils asynchronous behavior featured with phase mixing in the high-frequency components of sunspot activity and solar F10.7, which may explain the different activity properties of the photosphere and corona on a short time scale. The comprehensive explanations of wavelet squared coherency and cross-wavelet phase angle using Morlet wavelet based on continuous cross-wavelet transform are given in [39] and [34] and references therein. The main concern of this paper is to apply the advanced wavelet based technique for the analysis of sunspot area and sunspot data. This paper is subdivided a section 2:includes the observation data and method of analysis; through section 3:we have interpreted the results and finally in the section 4:conclusion.

\section{Observational Data and Method of Analysis}

\subsection{Data Set}

The daily values of sunspot number of the whole solar disk, northern and southern hemispheres of the Sun are published by the "Solar Influences Data Analysis Centre" (http://sidc.oma.be/sunspot-data/), a solar physics research division of the Royal Observatory of Belgium.

We have used the daily and total (both the Northern and Southern hemisphere) values of sunspot number and area (expressed in units of millionths of the solar hemisphere) time series prior to January 2008 to May 2013, covering the ascending and maximum phase of Solar Cycle 24. The sunspot area data, beginning in May 9, 1874 were compiled at Royal Greenwich Observatory from a small network of observatories and available online at http://www.solarscience.msfc.nasa.gov.

\subsection{Wavelet Transform Technique}

We studied the relative behavior of sunspot area and sunspot number using wavelet based cross correlation techniques. Wavelet analysis is a tool for analyzing localized variations of power spectra within a time series [40], [34]. The wavelet technique decomposes a one-dimensional time series into a two-dimensional time-frequency space. Therefore, this method determines not only the periodicities of the dominant modes of variability, but also shows how the modes vary in time. Moreover, the wavelet technique is suitable to detect a signal which is relatively weak and intermittent in nature [40], [41]. To take into account describe wavelet in following ways:

\subsubsection{Continuous Wavelet Transform}

Wavelet analysis involves a transform from a one dimensional time series to a diffuse two-dimensional timefrequency image for detecting the localized and quasiperiodic fluctuations by using the limited time span of the data [40], [42]. The CWT has edge artifacts because the wavelet is not completely localized in time. It is therefore useful to introduce a cone of influence (COI) in which edge effects cannot be ignored. Here we take the COI as the area in which the wavelet power caused by a discontinuity at the edge has dropped to $e^{-2}$ of the value of edge. It is used to satisfy the admissibility condition [43], [40], [34], [42]. The continuous wavelet transform of the time series is given by

$$
W_{\Psi}(s, t)=\frac{1}{\sqrt{s}} \int_{-\infty}^{\infty} f(t) \Psi^{*}\left(\frac{t-x}{s}\right) d t,
$$

Where $f(t)$ is the time series of the sunspot data, $\psi^{*}$ is the complex conjugate of continuous wavelet function $\psi$, $s>0$ the scaling factor controlling the dilation of the mother wavelet and $a$ is the translation parameter determining the shift of the mother wavelet. It is common to use the Morlet wavelet, which consists of a complex exponential, where, $t$ is the time, $s$ is the wavelet scale and $\omega_{o}$ is the non-dimension frequency function. It is given by function [43] as

$$
\Psi(t)=\pi^{-\frac{1}{4}} e^{i \omega_{0} \eta} e^{-\frac{\eta^{2}}{2}}
$$

Where is the frequency parameter that allows one to shift the frequency range for the investigation. When using wavelets for feature extraction purposes the Morlet wavelet (with $\omega_{o}=66$ ) is a good choice, since it provides a good balance between time and frequency localization. Fig.2 shows the continuous wavelet spectra of the daily sunspot number and area. There evidently are common features in the wavelet power of the time series of high power above the $95 \%$ confidence level. Both have a small scale periodicity (range from 16-32 days) about 27 days as the rotational rate of Sun. We therefore, restrict our further treatment to this wavelet, although the methods we present are generally applicable [44]. The idea behind the CWT is to apply as a bandpass filter to the time series. The wavelet stretched in time by varying its scale $(s)$, so that $\eta=s$. $t$, and normalizing it to have unit energy. Detailed technical information about the Morlet wavelet is available at the website 
http://atoc.colorado.edu/research/wavelets/wavelet2.html.

\subsubsection{Cross Wavelet Transform}

Cross-wavelet transform (XWT) is an extension of wavelet transform to expose their common power and relative phase in time-frequency space between two time series [42]. The XWT of two time series $x$ and $y$ is defined as $W^{x y}=W^{X} W^{y}$, where $*$ denotes complex conjugation. We further the crosswavelet power as $\left|W^{X Y}\right|$. The complex argument $\arg \left(W^{X Y}\right)$ can be interpreted as the local relative phase between $x$ and in time frequency space. The cross wavelet transform exposes regions with high common power and further reveals information about the phase relationship. If the two series are physically related we would expect a consistent or slowly varying phase lag that can be tested against mechanistic models of the physical process. The univariate wavelet power spectrum can be extended to compare sunspot area $x(s)$ and sunspot number $y(s)$. One can define the wavelet cross spectrum as the expectation value of the product of the corresponding

$$
W_{x y}(s, t)=W_{x}(s, t) W y^{*}(s, t)
$$

Where * denotes the complex conjugate and $W_{x}(s, t)$ $W_{x}(s, t)$ and $W_{y}(s, t)$ are the wavelet transform of $x(s)$ and $y(s)$ respectively. It not only determines the periodicities of the dominant modes of variability, but also shows how the modes vary in time. In contrast to the WPS, the WCS are complex valued. In order to get the phase relationship, we employed the codes provided by [34], which is displayed in Fig. 3. Furthermore, XWT shows that the two time series are in phase in the area, namely about 27 days periodic scale, with significant common features found in Fig. 2 with their confidence level is above 95\%. However, during the maximum (i.e from year 2011 to 2013) phase arrows are pointing in the right direction and it shows that both time series are synchrony. The high frequency components range from 4 to 8days scale demonstrates the strong phase mixing because arrows at high frequencies are randomly distributed. [45] found via cross-recurrence plots the high frequency components in hemispheric sunspot activity are not responsible for the strong phase synchronization.

\subsubsection{Wavelet Coherence}

The wavelet coherence (WTC) another useful measure, which can show coherent cross-wavelet transform, is in timefrequency space. WTC is necessary because the crosswavelet spectrum appears to be unsuitable for significant testing the interrelation between two processes [46]. Wavelet coherence is defined between two CWTs to find significant coherent even though the common power is low and WTC closely resembles a localized correlation coefficient in time frequency space [34]. The WTC between two time series $\mathrm{x}$ and $\mathrm{y}$ is defined as [34].

$$
R_{n s}^{2}(s)=\frac{\left|s\left(s^{-1} W_{n}^{x y}(s)\right)\right|^{2}}{s\left(s^{-1}\left|W_{n}^{x}\right|^{2}\right) * s\left(s^{-1}\left|W_{n}^{y}(s)\right|^{2}\right)}
$$

where $\mathrm{S}_{\text {scale }}$ is smoothing operator. The wavelet coherence can be taken as a correlation coefficient to show the localization of time frequency, as the definition and the traditional correlation coefficient are very smaller. In this context, the smoothing operator $\mathrm{S}$ is

$$
\mathrm{S}(\mathrm{W})=\mathrm{S}_{\text {scale }}\left(\mathrm{S}_{\text {time }}\left(\mathrm{W}_{\mathrm{n}}(\mathrm{S})\right),\right.
$$

where $S_{\text {time }}$ denotes smoothing along the wavelet scale axis, and $S_{\text {scale }}$ smoothing along the time axis. In the context of the Morlet wavelet, the smoothing operators given as [31]

$$
\begin{gathered}
\left.S_{\text {time }}(W)\right|_{S}=\left.\left(W_{n}(S) * C_{1}^{-\frac{t^{2}}{2 s^{2}}}\right)\right|_{s}, \\
\left.S_{\text {scale }} W\right|_{s}=\left.\left(W_{n}(S) * c_{2} \Pi(0.6 s)\right)\right|_{n}
\end{gathered}
$$

where $c_{1}$ and $c_{2}$ are normalization constant and $\Pi$ is a rectangle function. The factor 0.6 is a scale decorrelation length determined empirically for the Morlet wavelet [47]. Both convolutions are discrete. As a result, the normalization coefficients have to be determined numerically [34]. Fig. 4 displayed the result of the XWT between the SSA and SSN. The WTC represents the strong phase mixing at period scale around the 32-128 days over the time period 2009-2012. However, the WTC indicates the noisy behavior with strong phase mixing in the high frequency components of both the SSN and SSA.

\section{Results and Discussion}

It is started by applying the well known wavelet tools, developed by [40], on the daily grouped sunspot number and sunspot area data from January 2008 May 2013. The current Solar Cycle 24 rises at a lower level of activity during the ascending phase. Fig. 1 shows the variations in both the parameters. From the Fig.1 it is cleared that (i) SSN and SSA solar indices never peak at the same time, and the SSN peaks earlier than the SSA; (ii) however, the SSA and SSN develops with the same magnetic field variations on the photosphere of the Sun and the magnetic field strength increases according to the ascending epochs. An understanding of complexity in the periodicities of the SSA and SSN may provide insight into the complex dynamics of the solar magnetic field in the two hemispheres. 

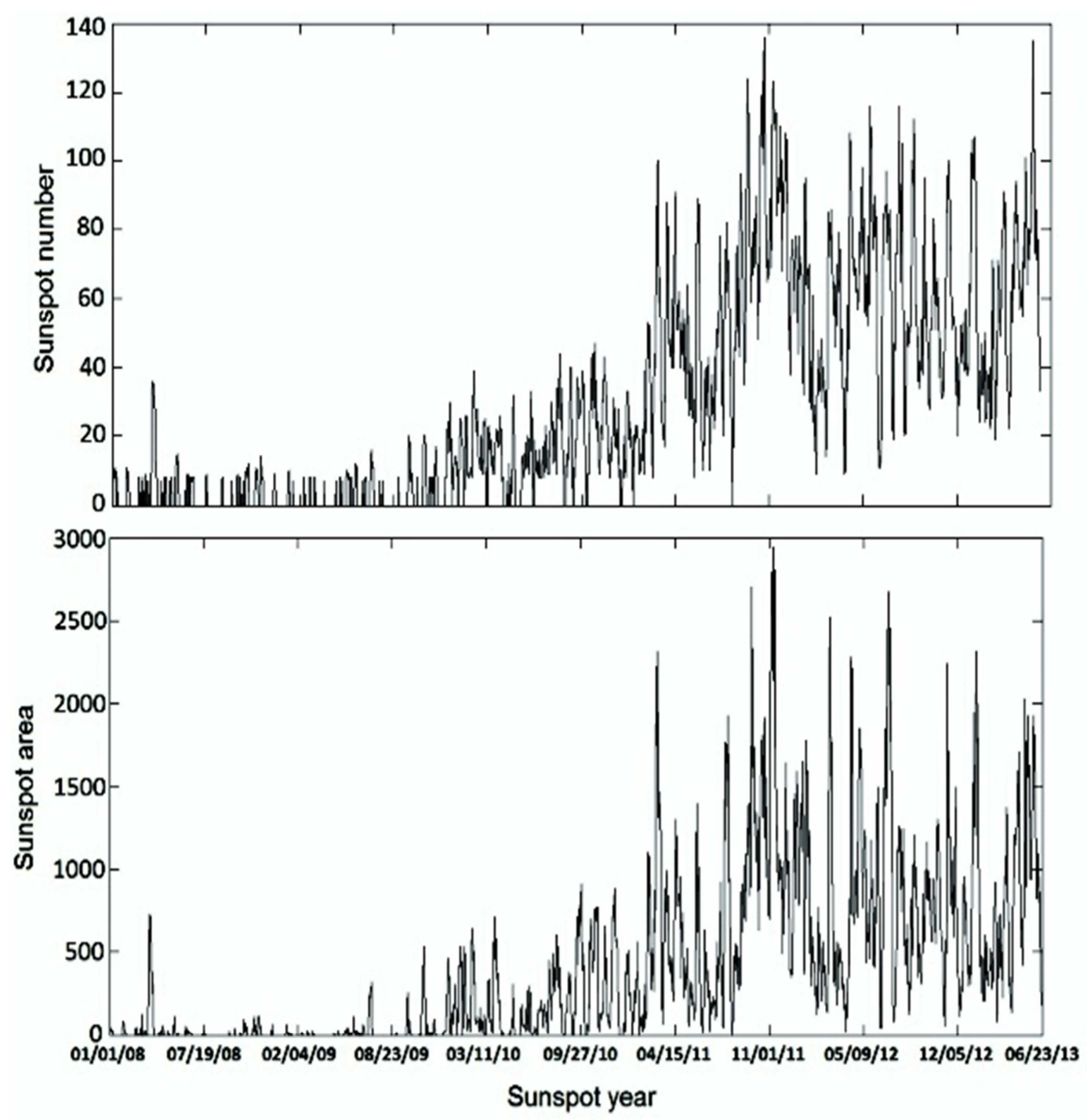

Fig. 1. The distribution of the sunspot number (upper panel) and sunspot area (bottom panel) for the time interval 2008 January to 2013 May.

The continuous wavelet power spectra for sunspot area and sunspot number are calculated and shown in Fig. 2 with a cone of avoidance and contours enclosing regions of greater than $95 \%$ confidence level. It was noticed that the spectral power is relatively weak at the short periods or at high frequencies. However, it is not difficult to locate some distinct dual-patches of relatively higher contours at around scale factor (i.e. periods) of around 16 to 64 days from September 2010 to May 2013. It is found that large variations at the time flow have a periodicity of around 27 day for both sunspot number and the sunspot area. The other small peaks cannot be considered from coincidence due to multiple testing [34].

The XWT and WTC are rendering the regions with high common power and make known information about the phase relationship between two time series. The WTC between SSN and SSA are shown in Fig. 4. Compared with the XWT a larger black contour stands out as being significant and all these area show phase relationship between SSA and SSN. The XWT and WTC have edge artifacts because the wavelet is not completely localized in time. It is therefore necessary to introduce a cone of influence (COI) in which the transformation suffers from these edge effects. The COI is defined so that the wavelet power for a discontinuity at the edges decreases by a factor $\mathrm{e}^{-2}$ [34]. The thin black contour (inside COI) demonstrates the periods above 95\% confidence level [40]. In these figures, arrows point to the right when processes are in phase and to the left when they are in anti-phase. If an arrows points up (down), then the first process leads (lags) these con done. The statistical significance of wavelet power can be assessed relative to the null hypothesis that the signal is generated by a stationary process with a given background power spectrum. The thick contour shown in figure designates the time series against red noise.

The XWT shows that almost all arrows point to the right and indicate 27 day periodic time in the period-scale. It means the sunspot number and sunspot area are in phase in this area. Statistically it is found that the significant result that there liable results are located within the cone of 
influence and the arrows beyond the cone of influence are not reliable. It is also noted that the arrows are fairly distributed in other periodic belts, implying strong phase mixing. The squared wavelet coherency is a measure of the intensity of the covariance of the two series in time-frequency space [40]. We have found that strong value of coherency in the period of 32-128 days of the epoch 2009-2012. All the sections show both the times series correlating to each other. By [48] shown that 27 days periodicity more pronounced during the descending phase than ascending phase of solar cycle. [49] have found the 27 days periodicity (with 13.5 days being its harmonic) in the dynamic parameters of the solar wind, Bx, By and the geomagnetic indices by both the wavelet analysis and the Lomb/Scargle periodogram. The rotational periodic of the Sun is expected to provide useful information about solar interior dynamics and mechanism of generation of the solar magnetic field and its emergence on the solar surface [16], [50]. [51] also predicted the values 112 of sunspot number for solar cycle 24, prior to 2011-2012 which is displayed in Fig.1 (upper panel). Solar cycle 24 has the lower amplitude than that of cycles 21, 22 and 23 which is consistent with the results obtained by [52] and [53]. [54] found the short-term periodicities in the daily data of the sunspot numbers and areas are investigated separately for the full disk, northern, and southern hemispheres during Solar Cycle 23 for a time interval from 1 January 2003 to 30 November 2007 corresponding to the descending and minimum phase of the cycle. The wavelet power spectrum technique exhibited a number of quasi-periodic oscillations in all the datasets. In the high frequency range, we find a prominent period of interval 22-35 days in both sunspot indicators.

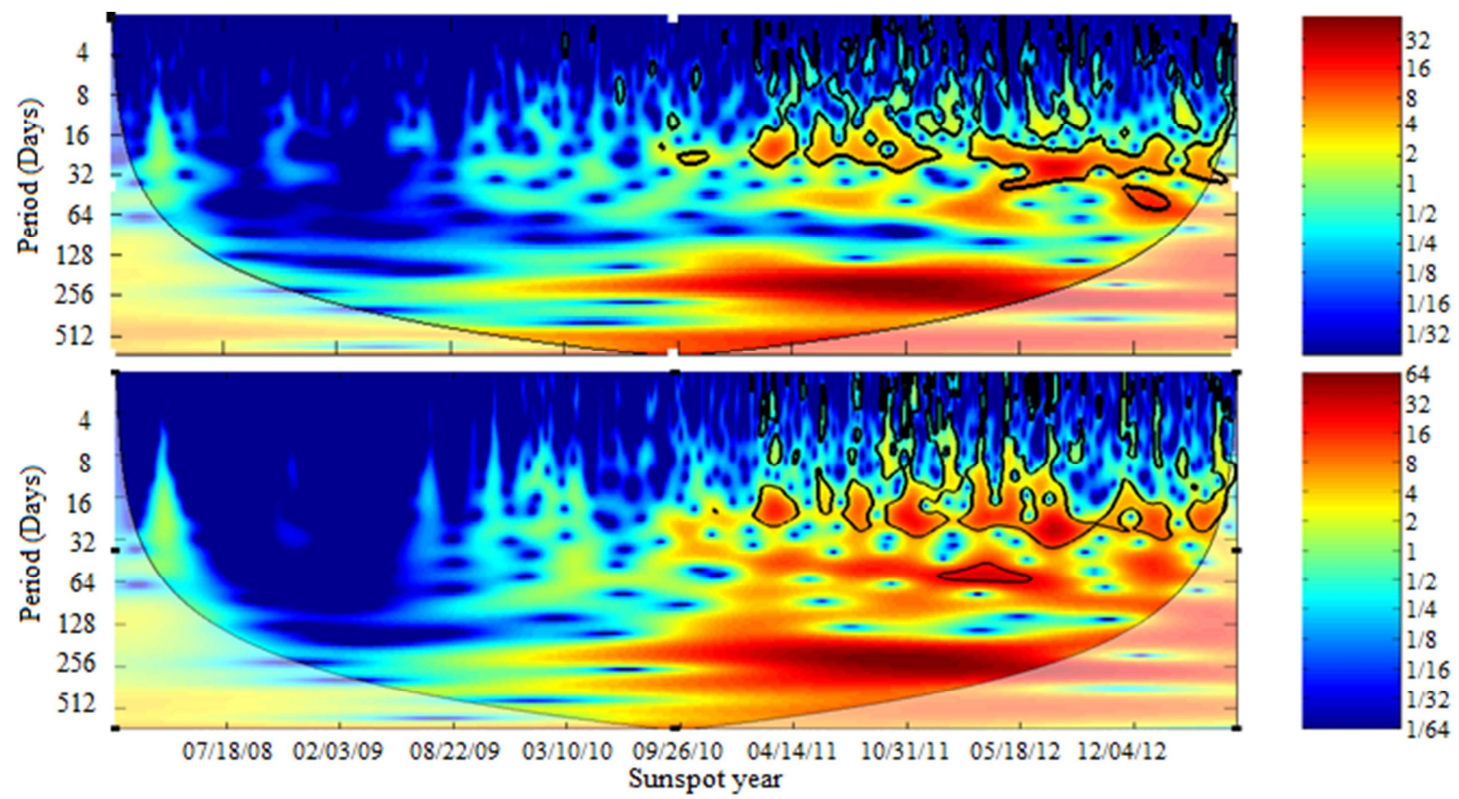

Fig. 2. Continuous Wavelet power spectra (WTC): Daily sunspot tnumber (upper panel: fig.1) and sunspot area (bottom panel: fig. 1), with a cone of avoidance and enclosed regions of greater than 95\% confidence level (thick black solid line).

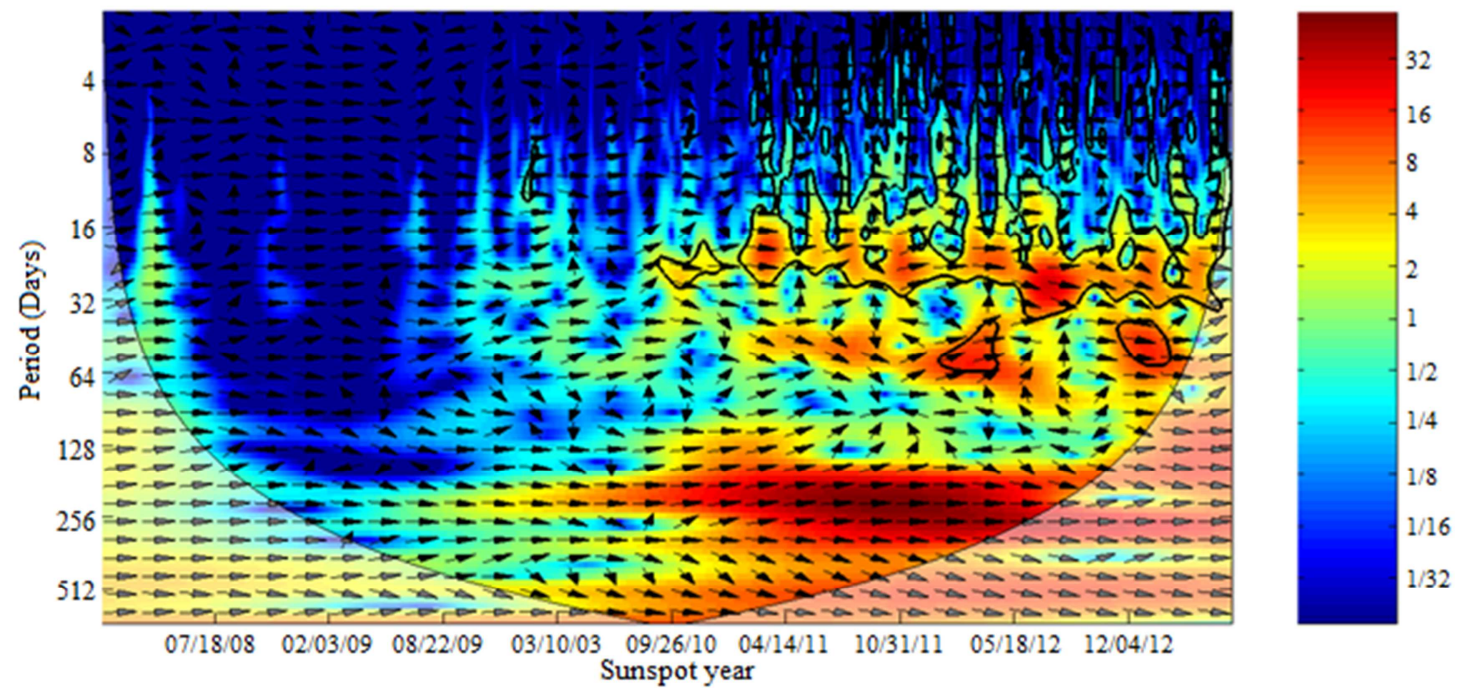

Fig. 3. Cross-wavelet spectra (XWT): Daily sunspot number and sunspot area with a cone of avoidance and enclosed regions of greater than 95\% confidence level (thick black solid line). 


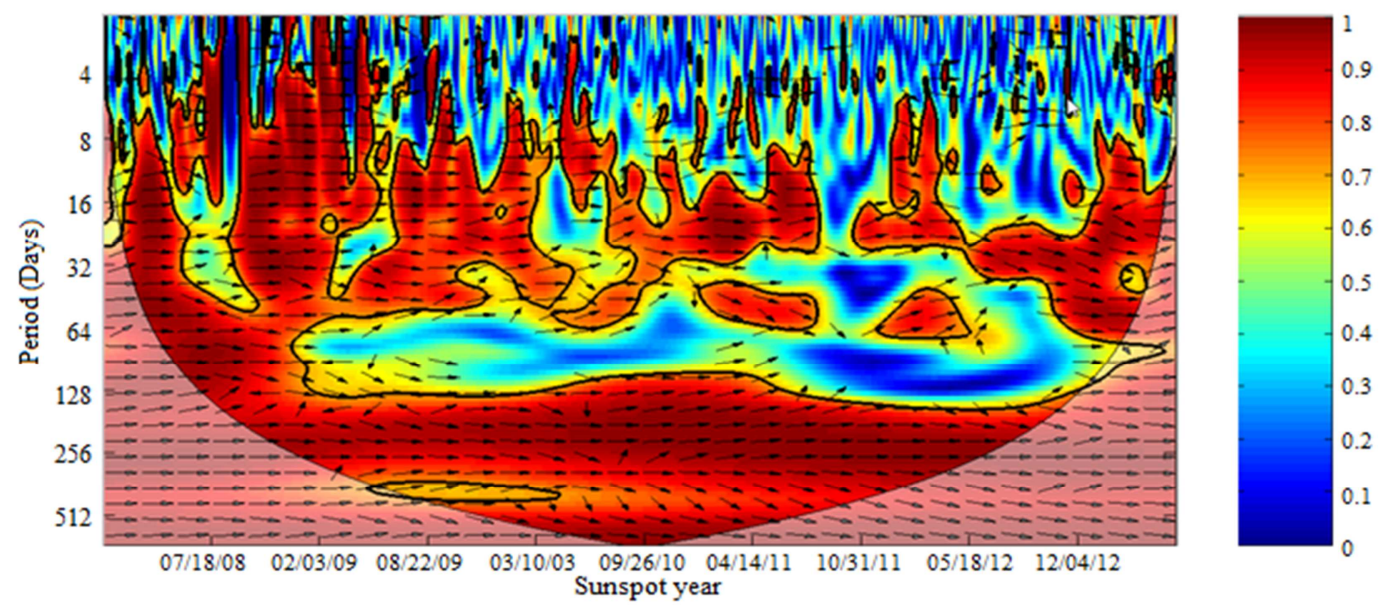

Fig. 4. Wavelet coherence of (WTC): Daily sunspot number and sunspot area with a cone of avoidance and enclosed regions of greater than $95 \%$ confidence level (thick black solid line).

\section{Conclusions}

This paper deals with the wavelet cross-correlation analysis of the daily values of SSA and SSN. Our study covers the entire maximum phase and a major portion of the minimum phase of Solar Cycle 24 (period from January 2008 to May 2013). For total sunspot number and sunspot area wavelet analysis detected a significant period of about 27 days (solar orbit rotation). However, this period varies from 16 to 64 days. This period is more stable than the other shortterm periods and appeared form September 2010 to May 2013. With this periodicity, we have concluded that the solar cycle 24 also include the most remarkable period during the ascending phase. Here, the many periodicities are not considered due to the maximum peaks are below the significance level. Near 27 day periodicity is noticed in th sunspot number [55], [56], [57] and in the sunspot area [57]. It is also detected in the different following solar activities $\mathrm{CaK}$ plage area [58], globally averaged coronal radio fluxes [59], [60], [61] and in the coronal mass eejection (CME) activity during Solar Cycle 23 [62]. [63] revealed the 27 days periodicity is present almost in their analysis using the wavelet technique on the daily data of sunspot numbers covering the Solar Cycles 10-23.

The results of this work clearly demonstrate that there exists a strong correlation around the year 2012. In conclusion we have found evidence for a 27 day periodicity in both the sunspot area and sunspot number during the ascending branch of solar cycle 24 and a very weak evidence for the fundamental period reported by [48]. This may arise because of different characteristics of the current cycle. For the exact conclusion we have to wait until the end of cycle 24. However, the strong magnetic fields in sunspots are not necessarily unstable. It is worth noting that the same time interval similar periodicities exist in the SSA and SSN. The continuous wavelet transforms of the two time series show that the 16-32 periodic range are statistically significance for short-term periodicities. The cross wavelet transform of the two time series shows that there is an area with high common spectral power, located at the 16-64 periodic belts, where the two time series are in phase. The cross wavelet transform (XWT) confirms the results given by wavelet coherence (WTC), but WTC is found more suitable to find a coherent oscillation of the two time series than XWT. This analysis reveals that the Solar Cycle 24 has only mid range periodicity which can predict that this cycle has minimum amplitude than others.

\section{Acknowledgments}

We thank to Solar Influence Data Analysis Center (SIDC) and NASA Solar Science Center for providing data. A Matlab software package being used by the authors for performing XWT and WTC can be found at http://www.pol.ac.uk/home/research/waveletcoherence/. One of us (SKK) is thankful to University Grants Commission, New Delhi (India) for financial assistance under the scheme of RGNF.

\section{References}

[1] Bartels, J. (1934). Terr. Magn. Atmos. Elec., 39, 201.

[2] Watari, S. (1996). Solar Phys. 168, 413.

[3] Polygiannakis, J., Preka-papadema, P., Petropoulos, B., Pothitakis, G., Moussas, X., pappas, G., Hilliaris, A. (2002). In: H. Swaya- Lacoste (ed.) SOLMAG2002. Proc. Magnetic Coupling of the Solar Atmosphere Euro conference, ESASP$505,537$.

[4] Katsavrias, C., Preka Papadema, P., Moussas, X. (2012). Wavelet Analysis on Solar Wind Parameters and Geomagnetic Indices. SolarPhys. DOI10.1007/s11207-012-0078-6.

[5] Lean, J. L., Brueckner, G. E. (1989). intermediate-term solar periodicities:100-500days, ApJ, 337, 568.

[6] Lean, J. L. (1990). Evolution of the 155 day periodicity in sunspot areas during solar cycles 12 to 21, Astrophys. J. 363, 718 . 
[7] Carbonell, M., Ballester, J. L. (1992). Astron. Astrophys. 255, 350 .

[8] Oliver, R., Carbonell, M. and Ballester, L. (1992). Sol. Phys., 137,141 .

[9] Krivova, N. A., Solanki, S. K. (2002). Astron. Astrophys.394, 701.

[10] Richardson, I. G. and Cane, H. V.: 2005, Geophys. Res. Lett., 32, Lo 2104.

[11] Atac, T., Ozguc, A., Rybak, J. (2006). Solar Phys. 237, 433.

[12] Joshi, B., Pant, P., Manoharan, P. K. (2006). Astron. Astrophys. 452, 647.

[13] Rieger, E., Share, G. H., Forrest, D. J., Kambach, G., Reppin, C., Chupp, E. L.(1984). Nature, 312, 623.

[14] Kiplinger, A. L., Dennis, B. R., Orwig, L. E. (1984). BAAS, 16,891

[15] Dennis, B. R. (1985). SolarPhys., 100, 465.

[16] Ichimoto, K., Kubota, J., Suzuki, M., Tohmura, I., Kurokawa, H. (1985). Nature, 316, 422.

[17] Delache, P. H., Laclare, F., Sadsoud, H. (1985). Nature, 317, 416.

[18] Bogart, R. S., Bai, T. (1985). ApJ, 299, L51.

[19] Bai, T., and Sturrock P. A. (1987). The 152 day periodicity of the solar flare occurrence rate, Nature, 327, 601-604.

[20] Ribes, E., Merlin, Ph., Ribes, J. C., Barthalot, R. (1987), Annales Geophysicae 7, 321.

[21] Lean, J. L., Bruckner, G. E. (1989). Intermediate-term solar periodicities: 100-500 days, APJ, 337, 568.

[22] Ozguç, A., Atac, T. (1989). Solar Phys., 123, 357.

[23] Carbonell, M., Ballester, J. L. (1990). A\&A, 238, 377.

[24] Droge, W., Gibbs, K., Grunsfeld, J. M., Meyer, P., Newport, B. J. (1990). ApJS, 73, 279.

[25] Pap, J., Tobiska, W. K., Bouwer, S. D. (1990). SolarPhys., $129,165$.

[26] Kile, J. N., Cliver, E. W. (1991). ApJ, 370, 442.

[27] Verma, V. K., Joshi, G. C. and Paliwal, D. C. (1992). Solar Phys., 138, 205.

[28] Cane, H. V., Richardson, I. G., von Rosenvinge, T. T. (1998). Geophys. Res. Lett., 25, 4437.

[29] Ballester, J. L., Oliver, R., Baudin, F. (1999). ApJ, 522, L153.

[30] Crowley, T. J., Berner, R. A. (2001). $\mathrm{CO}_{2}$ and climate change. Science 292, 870-872.

[31] Shaviv, N. J., Veizer, J. (2003). Celestial driver of Phanerozoic climate? GSA Today 13: 4-10.

[32] Royar, D. L. (2006). $\mathrm{CO}_{2}$ forced climate threshold during the Phanerozoic. Geochim Cosmochim Acta 70:5665-5675.

[33] Li, Q. X. (2008). Periodicity and hemispheric phase relationship in high-latitude solar activity. Sol. Phys. 249, 135.
[34] Grinsted, A., Moore J. C., Jevrejeva. S. (2004). Application of the cross wavelet transform and wavelet coherence to geophysical time series. Nonlinear Proc. Geophys., 11, 561-566.

[35] Davis, J. C. (2002). Statistics and data analysis I geology, $3^{\text {rd }}$ edn. Willey, NewYork, p 637.

[36] Morlet, J., Arens, G., Fourgean, E. and Giard, D. (1982). Wavepropagation and sampling theory, part1; comples signal land scattering in multi layer media. Journal of Geophys. 47:203-221.

[37] Zhang, Xue Feng, GuiMing, Le. And Zhang, Yan. Xia. (2012). Chinese Science Bulletin, Vol. 57, No. 2078-2082.

[38] Le, G. M. (2004). Wavelet analysis of the schwabe cycle properties in solar activity. Chin J Astron Astrophys, 4:578582.

[39] Torrence, C. and Webster, P. (1999). International changes in the ENSO-Monsoon System, J. Clim., 12, 2679-2690.

[40] Torrence, C. and Compo, G. P. (1998). A practical guide to wavelet analysis, Bull. Am. Meteorol. Soc., 79, 61-78.

[41] Knaack, R., Stenflo, J. O., S. V., Berdyugina: 2005, Astron. Astrophys. 438, 1067.

[42] Li, K. J., Gao, P. X., \& Su, T. W. (2005). Sol. Phys., 229, 181.

[43] Farge, M. (1992). Ann. Rev. Fluid Mech. 24, 395.

[44] Foufoula-Georgiou, E. and Kumar. P., (1995). Wavelet in Geophysics, Academic Press, 373.

[45] Zolotova, N. V., \& Ponyavin, D. I.(2006), A \& A, 449, L1.

[46] Maraun, D., Kurths, J. (2004). Nonlinearproc. Geophys., 11, 505 .

[47] Christopher, T., Gilbert, P. C. (1998). A practical guide to wavelet analysis. Bullamer Meteorlog Soc, 79, 61-68.

[48] Ozguç, A., Ataç, T. and Rybák, J. (2002) J. Geophys. Res. 107, 10. 1029/2001JA009080.

[49] Katsavrias, Ch., Preka-Papadema, P., Moussas, X. (2012). astro-ph. SR, arXiv:1205.2229v1.

[50] Sturock, P. A., Bai, T. (1992). Apj, 397, 337.

[51] Tong, Xu., Jian, Wu., Zhen-Sen, Wu., and Qiang, Li. (2007). Chin. J. Astron. Astrophys. Vol. 8, No. 3, 337-342.

[52] Duhau, S. (2003). Wavelet analysis of solar activity recorded by sunspot groups. Solar Phys. 213:203-212.

[53] Wang, J. L., Gong, J. C., Liu, S. Q., et al. (2002). The prediction of maximum amplitudes of solar cycles and the maximum amplitude of solar cycle 24. Chin. J. Astron. And Astrophys, 2 (6): 557-562.

[54] Chowdhury Parth. (2010). Short Term Periodicities in Sunspot Activities During the Descending Phase of Solar Cycle 23. Solar Phys 261:173-191. DOI10.1007/s11207-009-9478-7.

[55] Lean, J. L. (1991). Rev. Geophys., 29, 4, 505.

[56] Balthasar, H. (2007). A \& A, 471, 281.

[57] Kilick, H. (2009). Sol. Phys., 253, 281.

[58] Singh, Jand Prabhu T. P. (1985). Sol. Phys., 97, 203. 
[59] Kane, R. P., Vats, H. O. and Sawant, H. S. (2001). Sol. Phys., $2011,181$.

[60] Kane, R. P. (2002). Sol. Phys. 205, 351.

[61] Hiremath, K. M. (2002). In Proc. IAU Coll 188, (ESASP.505). P. 425.
[62] Lara, A, Borgazci, A, Mendas, Odim, Jr., Rosa, R. R., Dominguer, M. O. (2008). Solar Phys, 248, 155.

[63] Yin, Z. Q., Han, Y. B., Ma, L. H., Le, G. M., Han, Y. G. (2007). Chin. J. Astron. Astrophys. 7, 823. 